CLNS 1451, NSF-ITP-97-022, hep-th/9703085

\title{
Purely Transmitting Defect Field Theories
}

\author{
Robert Konik \\ Newman Laboratory \\ Cornell University \\ Ithaca, NY 14853 \\ André LeClair国 \\ Institute for Theoretical Physics \\ University of California \\ Santa Barbara, CA 93106-4030
}

We define an infinite class of integrable theories with a defect which are formulated as chiral defect perturbations of a conformal field theory. Such theories can be interacting in the bulk, and are purely transmitting through the defect. The examples of the sine-Gordon theory and Ising model are worked out in some detail.

$3 / 97$

1 On leave from Cornell University 


\section{Introduction}

The class of integrable quantum field theories has grown dramatically in the last several years due primarily to the idea of Zamolodchikov that defines such theories as perturbations of known conformal field theories [1]. This approach has been generalized to theories on the half-line with non-trivial boundary interactions [2].

Another class of theories with many interesting applications has a single impurity or defect not at the boundary but embedded in the sample. Such a theory can be described by the Euclidean action,

$$
S=S_{\mathrm{bulk}}+g \int d t \mathcal{O}(0, t)
$$

where the bulk action is the integral of the Lagrangian density, $S_{\text {bulk }}=\int d x d t \mathcal{L}$, and the spatial variable $x$ varies over the whole real line. The perturbation by the defect operator $\mathcal{O}$ at $x=0$ modifies the Hamiltonian as follows:

$$
H=H_{\mathrm{bulk}}+g \mathcal{O}(0)
$$

For a general defect operator $\mathcal{O}$, translation invariance is broken. Thus particles interacting with the defect can be both transmitted or reflected. The integrability of such defect field theories was studied in [3]. Unfortunately, non-trivial solutions to the Yang-Baxter-like constraints involving both reflection and transmission are severely limited: for diagonal bulk scattering, it was shown in [3] that non-trivial reflection and transmission are only allowed in theories that are free in the bulk. Thus integrable impurity problems that are interacting in the bulk are of only two types: purely reflecting, which is the same as a boundary theory, and purely transmitting.

In this paper, using conformal perturbation theory, we argue that the purely transmitting theories arise from actions of the kind (11.1) where the defect operator $\mathcal{O}$ is chiral. We describe how to associate an integrable chiral defect theory to every known integrable bulk perturbation of a conformal field theory. We show that if the bulk theory is a massless conformal field theory then the chiral defect theory can be mapped onto a massless boundary field theory. Interestingly, in general this map requires one to introduce defect degrees of freedom. Genuinely new models occur when the bulk is massive, since in this case the theory cannot be folded onto a known boundary theory. These general features are illustrated with the examples of the chiral defect sine-Gordon theory and a chiral defect perturbation of the Ising model.

2 We verified that the same occurs in a non-diagonal bulk scattering theory, specifically the sine-Gordon theory (though with no defect degrees of freedom). 


\section{Integrals of Motion and Conformal Perturbation Theory}

We study first the general features of integrals of motion in theories with a defect defined by an action (1.1). The defect separates space into two regions, $x<0$ and $x>0$, and one must distinguish the fields in these regions. For any field $\Psi(x, t)$,

$$
\begin{aligned}
& \Psi(x, t)=\theta(x) \Psi^{(+)}(x, t)+\theta(-x) \Psi^{(-)}(x, t) \\
& \Psi(0, t) \equiv \frac{1}{2}\left(\Psi^{(+)}(0, t)+\Psi^{(-)}(0, t)\right) .
\end{aligned}
$$

Suppose we are given a bulk conserved current $J_{\mu}$, satisfying $\partial_{t} J_{t}-\partial_{x} J_{x}=0$ where $x \neq 0$. This current will lead to a conserved charge in the presence of the defect if the following boundary condition is satisfied

$$
J_{x}^{(+)}(0, t)-J_{x}^{(-)}(0, t)=\partial_{t} \Theta(t)
$$

for some operator $\Theta$. A conserved charge satisfying $\partial_{t} Q=0$ is then constructed as follows:

$$
Q=\Theta(t)+\int_{-\infty}^{\infty} d x J_{t}
$$

We now take the bulk theory to be defined by a bulk perturbation of a conformal field theory (CFT):

$$
S_{\mathrm{bulk}}=S_{C F T}+\lambda \int d x d t \Phi(x, t) .
$$

The CFT must be a specific defect CFT, i.e. the boundary conditions at the defect must preserve the conformal invariance. See section 3. For a given CFT, certain perturbing fields $\Phi$ lead to an integrable quantum field theory [1]. We henceforth assume that based on the specific CFT of the bulk, $\Phi$ has been chosen to define an integrable theory. This implies there are an infinite number of conserved currents. In conformal perturbation theory, these currents have the following description. When $\lambda=0$, the perturbing field factorizes into left and right moving parts: $\Phi(z, \bar{z})=\phi_{L}(z) \phi_{R}(\bar{z})$, where $z=t+i x$, $\bar{z}=t-i x$. If the theory is integrable, there exists an infinite number of chiral fields (for $\lambda=0),\left\{J_{L}(z), J_{R}(\bar{z})\right\}$ with the following operator product expansion with the perturbing operator:

$$
\begin{aligned}
\phi_{L}(w) J_{L}(z) & =\ldots \frac{1}{w-z} \partial_{z} H_{L}(z)+\ldots \\
\phi_{R}(\bar{w}) J_{R}(\bar{z}) & =\ldots \frac{1}{\bar{w}-\bar{z}} \partial_{\bar{z}} H_{R}(\bar{z})+\ldots
\end{aligned}
$$


i.e. the residue of the simple pole is a total derivative. This ensures the conservation of the following charges:

$$
\begin{aligned}
& Q=\int d z J_{L}+\lambda \int d \bar{z}\left(H_{L} \phi_{R}\right) \\
& \bar{Q}=\int d \bar{z} J_{R}+\lambda \int d z\left(H_{R} \phi_{L}\right) .
\end{aligned}
$$

With the right choice of defect perturbation, the operator product expansion (2.5) can ensure integrals of motion in the defect theory. Some analysis of the integrals of motion shows that in general the choice of the local defect operator $\mathcal{O}=\Phi$ does not lead to an integrable theory ${ }^{3}$. However a chiral perturbation does. Namely, we consider the action

$$
S=S_{\mathrm{bulk}}+g \int d t \phi_{L}(0, t)
$$

where $S_{\text {bulk }}$ is as in (2.4), where $\Phi=\phi_{L} \phi_{R}$. (We can just as well perturb with $\phi_{R}$ rather than $\left.\phi_{L}.\right)$

There are subtle issues concerning the locality of a chiral perturbation since for a general CFT the integrable chiral fields have fractional Lorentz spin. We will address this issue for the sine-Gordon theory below. Alternatively, this problem can perhaps be cured by multiplying $\phi_{L}$ by a discrete degree of freedom which can be interpreted as a zero mode of a right-moving field. These discrete degrees of freedom may be the origin of the defect degrees of freedom introduced below. For instance, see the treatment of the free fermion in section 6 .

First set $\lambda=0$. To first order in perturbation theory one has,

$$
J_{L}^{(+)}(0, t)-J_{L}^{(-)}(0, t)=\lim _{\epsilon \rightarrow 0^{+}} g \int d t^{\prime} \phi_{L}\left(0, t^{\prime}\right)\left(J_{L}(\epsilon, t)-J_{L}(-\epsilon, t)\right) .
$$

Using the operator product expansions (2.5),

$$
\begin{aligned}
& J_{L}^{(+)}(0, t)-J_{L}^{(-)}(0, t)=4 \pi i g \partial_{t} H_{L} \\
& J_{R}^{(+)}(0, t)-J_{R}^{(-)}(0, t)=0 .
\end{aligned}
$$

This implies the existence of two conserved charges for every $J_{L, R}$ :

$$
\begin{aligned}
& Q_{L}=4 \pi g H_{L}+\int d x J_{L} \\
& Q_{R}=\int d x J_{R} .
\end{aligned}
$$

3 In some cases a change of basis and/or an intricate folding of the theory can relate it to an integrable boundary theory; see section 5 . 
In specific models, one can usually apply scaling arguments to rule out higher order corrections in $g$ that could spoil the conservation laws. In the massless case, the folding construction of the next section indicates that if the boundary version of the conserved charges are exact to first order in $g$, then so are the above charges in the defect theory.

For the case of the energy momentum tensor, the above analysis indicates that both left and right moving momentum and thus both the usual energy and momentum are conserved. This means that there can be no reflection of particles off the boundary, as this would violate momentum conservation. Therefore, integrable theories defined by (2.7) are purely transmitting, at least in the massless case, with generally non-trivial transmission S-matrices through the defect.

Now we consider the theory (2.7) with $\lambda \neq 0$. One might expect that since the bulk perturbation couples left with right movers, these theories will have both reflection and transmission, and if the bulk S-matrix is non-trivial, the remarks made in the introduction would suggest this theory cannot be integrable. To settle the issue, one must check that the additional bulk terms in (2.6) do not spoil the boundary condition (2.2). We will address these points in the sine-Gordon example below. There we will argue, though not conclusively, that the theory continues to be integrable for $\lambda \neq 0$, and will propose transmission S-matrices for the model.

\section{Folding for a massless bulk theory}

\subsection{Defect Conformal Field Theory}

In conformal field theory, boundary conditions at the defect which preserve the conformal symmetry satisfy

$$
\begin{aligned}
& T^{(+)}(0, t)-T^{(-)}(0, t)=0 \\
& \bar{T}^{(+)}(0, t)-\bar{T}^{(-)}(0, t)=0
\end{aligned}
$$

where $T(z), \bar{T}(\bar{z})$ are the chiral and anti-chiral components of the energy-momentum tensor. Using the analyticity one can in general fold this theory onto the half-line $x>0$ as follows. Define boundary fields for $x>0$ as follows:

$$
\begin{aligned}
T_{B}(x, t) & =T^{(+)}(x, t), & \bar{T}_{B}(x, t) & =T^{(-)}(-x, t), \\
\bar{T}_{B}^{\prime}(x, t) & =\bar{T}^{(+)}(x, t), & T_{B}^{\prime}(x, t) & =\bar{T}^{(-)}(-x, t) .
\end{aligned}
$$


The fields $T_{B}, T_{B}^{\prime}$ are functions of $t+i x$ whereas $\bar{T}_{B}, \bar{T}_{B}^{\prime}$ are functions of $t-i x$ for $x>0$. The boundary conditions (3.1) imply

$$
\begin{aligned}
& T_{B}(0, t)-\bar{T}_{B}(0, t)=0 \\
& T_{B}^{\prime}(0, t)-\bar{T}_{B}^{\prime}(0, t)=0,
\end{aligned}
$$

thus in the region $x>0$ one has two boundary conformal field theories which are decoupled, corresponding to $T_{B}$ verses $T_{B}^{\prime}$. Boundary conformal field theories were studied in [4]. We conclude that defect conformal field theories are characterized by the tensor product of two conformal boundary conditions. This fact was used for instance in [5].

\subsection{Chiral Perturbations}

When the bulk theory is massless, i.e. $\lambda=0$ in (2.4), then the defect theory (2.7) can be reformulated as a boundary theory on the half-line by the general folding procedure above. This has already been used in a number of contexts, e.g. [6] [7]. Here the situation is even simpler, since in the perturbed chiral defect theory, only left-moving fields are coupled to the defect. Thus, the boundary theory defined by $T_{B}^{\prime}, \bar{T}_{B}^{\prime}$ decouples from the defect. Let $\varphi_{L}^{( \pm)}$denote the components of an arbitrary chiral field $\varphi_{L}$ on either side of the defect, as in (2.1). Now, let us define a boundary theory in the region $x \geq 0$, where for each field $\varphi_{L}$ of the defect theory we associate the boundary theory fields $\varphi_{L, R}$ :

$$
\varphi_{L}(x, t)=\varphi_{L}^{(+)}(x, t), \quad \varphi_{R}(x, t)=\varphi_{L}^{(-)}(-x, t), \quad x>0 .
$$

Since the bulk is massless, $\varphi_{L}(x, t)=\varphi_{L}(z), \varphi_{R}(x, t)=\varphi_{R}(\bar{z})$. The boundary condition (2.9) now becomes in the boundary theory:

$$
J_{L}(0, t)-J_{R}(0, t)=4 \pi i g \partial_{t} H_{L} .
$$

The above condition assures the existence of a conserved charge in the boundary field theory [2]:

$$
Q=\left(\int_{0}^{\infty} d x\left(J_{L}+J_{R}\right)\right)+4 \pi g H_{L} .
$$

$Q$ is the direct map of the charge $Q_{L}$ of the defect theory to the boundary theory, i.e.

$$
Q_{L}=\int_{-\infty}^{0} d x J_{L}^{(-)}+\int_{0}^{\infty} d x J_{L}^{(+)}+4 \pi g H_{L}=Q .
$$

\section{Defect S-matrices}

Above we showed that interacting integrable theories with a defect are purely transmitting through the defect. We now derive the algebraic equations satisfied by the transmission S-matrices. The massive and massless cases should be treated differently. 


\subsection{Massive case}

Consider the bulk theory to be an integrable theory as in (2.4) with $\lambda \neq 0$, so that the spectrum consists of massive particles. Let $A_{a}(\theta)$ denote the formal FaddeevZamolodchikov operators for particles of type 'a' and rapidity $\theta$, where as usual the momentum is $m \sinh \theta$. We distinguish particles to the left (right) of the defect as $A_{a}^{(-)}\left(A_{a}^{(+)}\right)$. They are defined to satisfy the exchange relation

$$
A_{a}^{( \pm)}\left(\theta_{1}\right) A_{b}^{( \pm)}\left(\theta_{2}\right)=S_{a b}^{d c}\left(\theta_{1}-\theta_{2}\right) A_{c}^{( \pm)}\left(\theta_{2}\right) A_{d}^{( \pm)}\left(\theta_{1}\right)
$$

where $S_{a b}^{d c}$ is the bulk S-matrix satisfying Yang-Baxter, crossing and unitarity equations. Let us as before place the defect at $x=0$ and then introduce a defect operator $D_{\alpha}$, with defect degrees of freedom $\alpha$ taking values in some set. The scattering of particles through the defect is then described by the following exchange relations:

$$
\begin{aligned}
D_{\alpha} A_{a}^{(+)}(\theta) & =T_{\alpha a}^{\beta b}(\theta) A_{b}^{(-)}(\theta) D_{\beta} \\
A_{a}^{(-)}(\theta) D_{\alpha} & =\widetilde{T}_{a \alpha}^{b \beta}(-\theta) D_{\beta} A_{b}^{(+)}(\theta) .
\end{aligned}
$$

The transmission scattering matrices $T$ and $\widetilde{T}$ are not necessarily identical because parity symmetry is broken.

Consistency of (4.2) implies the unitarity condition

$$
T_{\alpha a}^{\beta b}(\theta) \widetilde{T}_{b \beta}^{c \gamma}(-\theta)=\delta_{a}^{c} \delta_{\alpha}^{\gamma}
$$

We will also require the crossing symmetry relation

$$
\widetilde{T}_{a \alpha}^{b \beta}(\theta)=T_{\alpha \bar{b}}^{\beta \bar{a}}(i \pi-\theta)
$$

where $\bar{a}$ is the charge conjugate of $a$.

Associativity of the algebra (4.2) demands $T$ and $\widetilde{T}$ satisfy the defect Yang-Baxter equations (DYB):

$$
\begin{gathered}
S_{a b}^{d c}\left(\theta_{12}\right) T_{\alpha c}^{\beta e}\left(\theta_{2}\right) T_{\beta d}^{\gamma f}\left(\theta_{1}\right)=T_{\alpha a}^{\beta c}\left(\theta_{1}\right) T_{\beta b}^{\gamma d}\left(\theta_{2}\right) S_{c d}^{f e}\left(\theta_{12}\right) \\
S_{a b}^{d c}\left(\theta_{12}\right) \widetilde{T}_{d \alpha}^{e \beta}\left(-\theta_{1}\right) \widetilde{T}_{c \beta}^{f \gamma}\left(-\theta_{2}\right)=\widetilde{T}_{b \alpha}^{c \beta}\left(-\theta_{2}\right) \widetilde{T}_{a \beta}^{d \gamma}\left(-\theta_{1}\right) S_{d c}^{e f}\left(\theta_{12}\right)
\end{gathered}
$$

where $\theta_{12}=\theta_{1}-\theta_{2}$. If $S_{a b}^{d c}=S_{b a}^{c d}$, then upon relabeling $\theta_{1} \rightarrow-\theta_{2}, \theta_{2} \rightarrow-\theta_{1}$, one sees that $\widetilde{T}_{a \alpha}^{b \beta}$ satisfies the same DYB equation as $T_{\alpha a}^{\beta b}$. 
A final constraint arises from hermiticity of the Hamiltonian. The transmission Smatrices are understood as the matrix elements

$$
\begin{aligned}
T_{\alpha a}^{\beta b}(\theta) & ={ }_{\text {out }}^{L}\langle\theta, b, \beta \mid \theta, a, \alpha\rangle_{\text {in }}^{R} \\
\widetilde{T}_{a \alpha}^{b \beta}(-\theta) & ={ }_{\text {out }}^{R}\langle\theta, b, \beta \mid \theta, a, \alpha\rangle_{\text {in }}^{L}
\end{aligned}
$$

where here $L, R$ denotes to the left or right of the defect. Hermiticity of the Hamiltonian demands

$$
\widetilde{T}_{a \alpha}^{b \beta}(\theta)=\left(T_{\beta b}^{\alpha a}(-\theta)\right)^{*}
$$

\subsection{Massless case}

Now we take the bulk theory to be massless, i.e. $\lambda=0$ in (2.4). Only left-moving particles couple to the defect, so we only deal with these left-movers and denote them $A_{a}^{L}(k)$, for $k<0$. It is convenient to parameterize the momentum with a rapidity $\theta$, $k_{L}=-\frac{\mu}{2} e^{-\theta}$, where $\mu$ is some scale. The scattering with the defect is described by

$$
D_{\alpha} A_{a}^{L}(\theta)=T_{\alpha a}^{\beta b}(\theta) A_{b}^{L}(\theta) D_{\beta},
$$

and the DYB equation is the same as (4.5a). The massless bulk scattering is described by

$$
A_{a}^{L}\left(\theta_{1}\right) A_{b}^{L}\left(\theta_{2}\right)=S_{a b}^{d c}\left(\theta_{1}-\theta_{2}\right) A_{c}^{L}\left(\theta_{2}\right) A_{d}^{L}\left(\theta_{1}\right)
$$

In the last section, for the massless case we showed at the level of fields how the problem can be mapped onto a boundary field theory. We now describe how this folding is performed in the scattering theory. Consider a massless boundary field theory on the half-line $x \geq 0$. This theory has both left and right moving particles. Parameterize the left-moving momenta as $k_{L}=-\frac{\mu}{2} e^{-\theta_{L}}$, and the right-moving momenta as $k_{R}=\frac{\mu}{2} e^{\theta_{R}}$, and introduce operators $A_{a}^{L}\left(\theta_{L}\right)$ and $A_{a}^{R}\left(\theta_{R}\right)$. (We will not display the subscripts $L, R$ on $\theta_{L, R}$ when the meaning is clear.) The scattering in the bulk is as in (4.9), and the same equation also holds for $A^{L} \rightarrow A^{R}$. By scale invariance, the $L-R$ scattering is independent of momentum. However non-trivial constant scattering is allowed, and will be important in the sequel. Thus we suppose

$$
A_{a}^{R}\left(\theta_{1}\right) A_{b}^{L}\left(\theta_{2}\right)=B_{a b}^{d c} A_{c}^{L}\left(\theta_{2}\right) A_{d}^{R}\left(\theta_{1}\right)
$$


where $B_{a b}^{c d}$ is a matrix of constants satisfying a braiding relation. The interaction with the boundary is encoded in a reflection matrix. Assuming the boundary to have no degrees of freedom, we introduce a boundary operator $\mathcal{B}$ satisfying:

$$
\mathcal{B} A_{a}^{L}(\theta)=R_{a}^{b}(\theta) \mathcal{B} A_{b}^{R}(-\theta)
$$

Consistency of (4.9) and (4.11) demands the massless boundary Yang-Baxter (BYB) equation:

$$
R_{a}^{c}\left(\theta_{1}\right) B_{c b}^{c^{\prime} b^{\prime}} R_{b^{\prime}}^{d}\left(\theta_{2}\right) S_{d c^{\prime}}^{e f}\left(\theta_{12}\right)=S_{a b}^{d c}\left(\theta_{12}\right) R_{c}^{e^{\prime}}\left(\theta_{2}\right) B_{e^{\prime} d}^{e d^{\prime}} R_{d^{\prime}}^{f}\left(\theta_{1}\right)
$$

When the L-R scattering is trivial, i.e. if $B_{a b}^{a^{\prime} b^{\prime}}=\delta_{a}^{a^{\prime}} \delta_{b}^{b^{\prime}}$, then the massless BYB equation (4.12) is identical to the DYB equation with no defect degrees of freedom. Namely, with no defect degrees of freedom, $T_{\alpha a}^{\beta b}=T_{a}^{b}$, and a solution to (4.12) is a solution to (4.5a) with $T_{a}^{b}(\theta)=R_{a}^{b}(\theta)$. Thus, a massless defect theory can be mapped onto a massless boundary theory by identifying the transmission and reflection S-matrices only when L-R scattering is trivial.

In general, one does not have the freedom to set the L-R scattering to 1 . Given a massive theory in the bulk, with $k=m \sinh \theta$, the massless limit $m \rightarrow 0$ is defined by letting $\theta=\theta_{L}-\alpha$, for left movers, and $\theta=\theta_{R}+\alpha$ for right-movers, and in both cases letting $\alpha \rightarrow \infty$ keeping $m e^{\alpha}=\mu$ held fixed. If $S_{a b}^{d c}(\theta)$ is the massive S-matrix, then in this limit $L-L$ and $R-R$ scattering are both given by the same $S$, whereas $L-R$ scattering is given by

$$
B_{a b}^{d c}=S_{a b}^{d c}(-\infty)
$$

Given a non-trivial $B$, one can try to establish a map between massless defect and boundary theories by introducing defect degrees of freedom. Indeed, in the example of the sineGordon theory treated in the next section, we find that by properly introducing defect degrees of freedom, we can find a one-to-one correspondence between solutions of DYB and massless boundary Yang-Baxter equations.

In light of this, we can propose a general approach to the massive case of purely transmitting defect theories. In the massive case, folding is not possible. For instance, in the massive case, the BYB equation reads [2],

$$
\widehat{R}_{a}^{c}\left(\theta_{2}\right) S_{b c}^{b^{\prime} c^{\prime}}\left(\theta_{1}+\theta_{2}\right) \widehat{R}_{b^{\prime}}^{d}\left(\theta_{1}\right) S_{c^{\prime} d}^{f e}\left(\theta_{12}\right)=S_{b d}^{c a}\left(\theta_{12}\right) \widehat{R}_{c}^{e^{\prime}}\left(\theta_{1}\right) S_{d e^{\prime}}^{d^{\prime} e}\left(\theta_{1}+\theta_{2}\right) \widehat{R}_{d^{\prime}}^{f}\left(\theta_{2}\right),
$$

where $\widehat{R}_{a}^{b}$ is the massive reflection S-matrix. It is clear that only in the massless limit (4.12) is there a chance of mapping BYB onto DYB. Our approach to the massive case will be as 
follows. Take the massless limit of the defect theory, and map it onto a known solution of a massless boundary field theory. If the $L-R$ scattering of latter is non-trivial, the map can only be established by introducing defect degrees of freedom. Finally, assume the massive case has the same defect degrees of freedom, and solve the defect Yang-Baxter equation for the massive case. We will illustrate this in the next section with the sine-Gordon theory.

\section{Defect sine-Gordon theory}

In this section we treat the chiral defect perturbation of the sine-Gordon theory, defined by

$$
S=\int_{-\infty}^{\infty} d x d t\left(\frac{1}{2}\left(\partial_{\mu} \Phi\right)^{2}+\lambda \cos \beta \Phi\right)+g \int d t \cos \beta \phi_{L}(0, t),
$$

where $\phi_{L}$ is the left-moving component of the scalar field, $\Phi=\phi_{L}+\phi_{R}$. The bulk sineGordon theory has a well-known infinite set of local conserved charges with spin equal to an odd integer. The general arguments of section 2 indicate that the above chiral defect sine-Gordon theory, at least in the massless limit $\lambda=0$, is also integrable.

At the level of the action (5.1) one can fold the massless theory as described in section 3 and explicitly obtain the boundary sine-Gordon theory (BSG). From the components $\phi_{L}^{( \pm)}$ of the field $\phi_{L}$ in the defect theory, define the boundary theory fields $\varphi_{L}(x, t)=\phi_{L}^{(+)}(x, t)$, $\varphi_{R}(x, t)=\phi_{L}^{(-)}(-x, t)$ for $x>0$, as in (3.4). In (5.1) since $\phi_{L}(0, t)=\left(\phi_{L}^{(+)}(0, t)+\right.$ $\left.\phi_{L}^{(-)}(0, t)\right) / 2$, in the map to the boundary theory one finds

$$
\int d t \cos \beta \phi_{L}(0, t) \rightarrow \int d t \cos \left(\beta\left(\varphi_{L}(0, t)+\varphi_{R}(0, t)\right) / 2\right)=\int d t \cos \left(\beta \Phi_{B} / 2\right),
$$

where now the boundary field $\Phi_{B}=\varphi_{L}+\varphi_{R}$ is the boundary sine-Gordon field. The complete action (5.1) becomes the BSG theory, as defined in [2]. We thus argue that in the massless case, if the boundary sine-Gordon conserved currents are exact to first order in $g$, then so are the conserved currents in the defect theory.

The problem with the apparent non-locality of the chiral perturbation in (5.1) can be resolved in the following way. Chiral conformal fields satisfy the braiding relations:

$$
e^{i a \phi_{L}(x, t)} e^{i b \phi_{L}(y, t)}=e^{ \pm i a b / 4} e^{i b \phi_{L}(y, t)} e^{i a \phi_{L}(x, t)} \quad \text { for }\left(\begin{array}{c}
x>y \\
x<y
\end{array}\right)
$$

Writing $\phi_{L}(0, t)=\lim _{\epsilon \rightarrow 0}\left(\phi_{L}(\epsilon, t)+\phi_{L}(-\epsilon, t)\right) / 2$, one finds that any field $\exp \left(i a \phi_{L}(0, t)\right)$ at the defect is local with respect to the perturbation $\exp \left( \pm i \beta\left(\phi_{L}(\epsilon, t)+\phi_{L}(-\epsilon, t)\right) / 2\right)$, 
since from the braiding relation (5.3), the phase from $\phi_{L}(\epsilon, t)$ is canceled by the phase from $\phi_{L}(-\epsilon, t)$.

Finally, it is interesting to consider taking not a chiral defect perturbation, but a local one in (5.1). Namely, let us replace $\cos \beta \phi_{L}$ with $\cos (\beta \Phi / 2)$, where $\Phi=\phi(z)+\bar{\phi}(\bar{z})$ is the local SG field. By making the change of basis $\phi_{ \pm}(x, t)=(\phi(x, t) \pm \bar{\phi}(-x, t)) / 2$, the interaction is seen to depend only on $\exp \left(\beta \phi_{+}\right)$. As $\phi_{ \pm}$are chiral, $\exp (\beta \Phi / 2)$ is integrable if $\exp (\beta \phi)$ is integrable. We can also see this via a folding into a boundary theory. As described in section 3 , when $g=0$ this theory can be folded onto two decoupled boundary conformal field theories for $x>0$. Namely, following (3.2), define boundary fields $\varphi_{L}(x, t)=\phi^{(+)}(x, t), \varphi_{R}(x, t)=\phi^{(-)}(-x, t), \varphi_{L}^{\prime}(x, t)=\bar{\phi}^{(-)}(-x, t), \varphi_{R}^{\prime}(x, t)=\bar{\phi}^{(+)}(x, t)$. Unlike the purely chiral perturbation (5.1), now the defect interaction couples the two boundary conformal field theories:

$$
\cos (\beta \Phi(0, t) / 2) \rightarrow \cos \left(\beta\left(\Phi_{B}+\Phi_{B}^{\prime}\right) / 4\right),
$$

where now $\Phi_{B}=\varphi_{L}+\varphi_{R}$, and $\Phi_{B}^{\prime}=\varphi_{L}^{\prime}+\varphi_{R}^{\prime}$. Since the combination $\Phi_{B}-\Phi_{B}^{\prime}$ decouples from the defect, what remains is again a single boundary SG theory. So again a local defect perturbation is seen to be integrable, but now via an intricate folding. Of course, these arguments only hold for $\lambda=0$.

\subsection{Scaling Analysis of Integrability}

We denote the set of charges for the sine-Gordon by

$$
\begin{aligned}
& Q_{s}=\int d x J_{s}-\lambda \int d x R_{s}, \\
& \bar{Q}_{s}=\int d x \bar{J}_{s}-\lambda \int d x \bar{R}_{s}
\end{aligned}
$$

where $J_{s}\left(\bar{J}_{s}\right)$ has spin s (-s), s even. The local fields $J_{s}, R_{s}, \bar{J}_{s}$, and $\bar{R}_{s}$ satisfy

$$
\begin{aligned}
& \partial_{\bar{z}} J_{s}=\lambda \partial_{z} R_{s}, \\
& \partial_{z} \bar{J}_{s}=\lambda \partial_{\bar{z}} \bar{R}_{s} .
\end{aligned}
$$

In the presence of a defect line at $x=0$, the most general modification the left moving fields, $J_{s}$ and $R_{s}$, can undergo is

$$
\begin{aligned}
J_{s}^{(+)}(0)-J_{s}^{(-)}(0) & =\sum_{n=1}^{\infty} g^{n} J_{s}^{n}, \\
R_{s}^{(+)}(0)-R_{s}^{(-)}(0) & =\sum_{n=1}^{\infty} g^{n} R_{s}^{n} .
\end{aligned}
$$


(We will assume that no terms dependent on $\lambda$ appear on the RHS of (5.7).)

The possible non-zero terms on the RHS of (5.7) can be partially determined by a scaling analysis. The scaling dimension of $g$ is $d(g)=1-\widehat{\beta}^{2} / 2, d\left(J_{s}\right)=s$, and $d\left(R_{s}\right)=$ $s-2+\widehat{\beta}^{2}$. (For convenience we have defined $\widehat{\beta}=\beta / \sqrt{4 \pi}$.) Since $J_{s}^{n}$ arises to n-th order in perturbation theory, it must come from the operator product expansion of $J_{s}$ with $\cos ^{n}\left(\beta \phi_{L}\right)$. Thus $d\left(J_{s}^{n}\right)=d\left(e^{i r \beta \phi_{L}} \mathcal{O}_{s}\right)$, where $r$ is an integer with $|r| \leq n$, and $\mathcal{O}_{s}$ is a local field of integer dimension $l$. Thus, $d\left(J_{s}^{n}\right)=l+r^{2} \widehat{\beta}^{2} / 2$. Matching dimensions in (5.7) one has $s=\left(r^{2}-n\right) \widehat{\beta}^{2} / 2+n+l$. For $\widehat{\beta}^{2}$ irrational, one needs $n=r^{2}$, and $s=r^{2}+l$. For the energy-momentum tensor at $s=2$, the only solution is $n=l=1$. Thus, the first equation in (2.9) is exact. For $s=4,6, \ldots$, higher order corrections in $g$ are not ruled out by scaling. However, since these higher order corrections are an issue in the massless theory, which can be folded onto the boundary theory which is known to be integrable, we can conclude that these possible higher order corrections do not spoil the conservation of $Q_{s}$.

The possible dimensions of the fields $R_{s}^{n}$ take the form $d\left(e^{i r^{\prime} \beta \phi_{L} \pm i \beta \phi_{R}} \mathcal{O}_{s}^{\prime}\right)$, where $r^{\prime}$ is an integer with $\left|r^{\prime}\right| \leq n+1$, and the local operator $\mathcal{O}_{s}^{\prime}$ has dimension $l^{\prime}$. Again matching dimensions in (5.7), one has $s=\left(r^{\prime 2}-n-1\right) \widehat{\beta}^{2} / 2+n+l^{\prime}+2$. For $\widehat{\beta}^{2}$ irrational, this requires $n+1=r^{\prime 2}$ and $s=r^{\prime 2}+l^{\prime}+1$. For $s=2$, the only solution is $n=0$, and thus $R_{2}^{(+)}-R_{2}^{(-)}=0$. For $s=4$, there is also no solution apart from $n=0$. For $s>4$, higher order corrections are possible.

A similar analysis for the right moving charges $\bar{Q}_{s}$ indicates that they are all unperturbed by the defect, i.e. $\bar{J}_{s}^{(+)}(0)-\bar{J}_{s}^{(-)}(0)=\bar{R}_{s}^{(+)}(0)-\bar{R}_{s}^{(-)}(0)=0$.

To summarize, in taking $\widehat{\beta}^{2}$ irrational, and accepting the folding argument by assuming that higher order corrections in $g$ do not spoil the conservation condition (2.9) of the massless theory, scaling arguments ensure that the bulk perturbation for $\lambda \neq 0$ does not spoil the conservation of $Q_{s}$ for at least $s=2,4$, whereas all $\bar{Q}_{s}$ remain conserved. For the energy-momentum tensor, one has the exact equations

$$
\begin{aligned}
& T^{(+)}(0)-T^{(-)}(0)=4 \pi i g \partial_{t} \cos \beta \phi_{L}, \\
& \bar{T}^{(+)}(0)-\bar{T}^{(-)}(0)=0
\end{aligned}
$$

while $R_{2}$ and $\bar{R}_{2}$ terms are continuous across the defect. Thus both energy and momentum are conserved. Though the above scaling analysis has some gaps (we have assumed no $\lambda$ terms in (5.7)) and doesn't prove the integrability of the defect theory when $\lambda \neq 0$, in the 
sequel we will assume that the theory (4.1) is integrable for any $\lambda$ and $g$ and is purely transmitting, though we emphasize we have not proven this conclusively.

At rational $\widehat{\beta}^{2}$ the above picture can be modified. In particular at $\widehat{\beta}^{2}=1$, the free fermion point, $R_{2}^{(+)}(0)-R_{2}^{(-)}(0)$ can have non-zero contributions at $n=1$ with $r^{\prime}=l^{\prime}=0$. Thus at the free fermion point left-moving momentum is not conserved, and this allows for reflection in addition to transmission; this is precisely what is found in section 6 . This is consistent with the remarks made in the introduction, since here the bulk S-matrix is -1 , and this allows both reflection and transmission.

\subsection{S-matrices}

We now describe the solution of the DYB equation in the case of chiral sine-Gordon. The S-matrix for solitons of $U(1)$ charge \pm 1 is as follows:

$$
\begin{aligned}
& S_{++}^{++}(\theta)=S_{--}^{--}(\theta)=a(\theta), \\
& S_{+-}^{+-}(\theta)=S_{-+}^{-+}(\theta)=b(\theta), \\
& S_{+-}^{-+}(\theta)=S_{-+}^{+-}(\theta)=c(\theta),
\end{aligned}
$$

where

$$
\begin{aligned}
a(\theta)=\sin (\gamma(\pi+i \theta)) \rho(\theta), \quad b(\theta) & =-\sin (i \gamma \theta) \rho(\theta), \quad c(\theta)=\sin (\gamma \pi) \rho(\theta), \\
\gamma & =\frac{8 \pi}{\beta^{2}}-1,
\end{aligned}
$$

and $\rho$ can be found in [8]. We will consider the massless and massive cases separately.

\subsubsection{Massless Case}

Consider first the massless case $\lambda=0$. The $L-R$ scattering, as defined by (4.13) is given by

$$
B_{a b}^{d c}=\delta_{a}^{d} \delta_{b}^{c} q^{-a b / 2}
$$

where

$$
q=-e^{-i \gamma \pi}
$$

As described in the last section, we first map the theory onto the massless boundary sine-Gordon theory. The reflection S-matrices $R_{a}^{b}$ are the massless limit of the GhoshalZamolodchikov solution [2], as described in [9], and as such, they satisfy (4.12) with $B_{a b}^{d c}$ given by (5.12). 
In order to describe the scattering theory of the defect theory, one must answer the question: what defect degrees of freedom are required in order that there is a one-to-one correspondence between solutions of the massless BYB (4.12) and the DYB (4.5a)? A similar question was posed in [10]. We introduce a defect degree of freedom $\alpha \in \alpha_{0}+Z$, where $\alpha_{0}$ is arbitrary. We require the defect transmission matrix $T_{\alpha a}^{\beta b}$ to satisfy $U(1)$ charge conservation $\alpha+a=\beta+b$, so that $\alpha$ can be interpreted as a $U(1)$ charge of the defect. One can show that either of the two choices

$$
\begin{aligned}
& T_{\alpha a}^{\beta b}(\theta)=q^{\alpha a / 2} R_{a}^{b}(\theta), \quad a+\alpha=b+\beta, \\
& T_{\alpha a}^{\beta b}(\theta)=q^{-\alpha a / 2} R_{a}^{-b}(\theta), \quad a+\alpha=b+\beta,
\end{aligned}
$$

where $R_{a}^{b}$ are the reflection S-matrices of the massless boundary sine-Gordon theory automatically is a solution of the DYB equation if $R$ satisfies (4.12). We will propose the second relation of (5.14) as the solution to the massless defect sine-Gordon theory. We do so because we expect $\mathrm{U}(1)$ charge to be violated in the folding process, i.e. for a free boundary theory $(g=0)$ we have

$$
R_{+}^{+}=R_{-}^{-}=0, \quad R_{+}^{-}=R_{-}^{+}=1,
$$

(there is maximal $\mathrm{U}(1)$ violation), while for a free defect theory, we expect solitons to pass through the defect charge unchanged.

\subsubsection{Massive Case}

Now we take the bulk to be massive $(\lambda \neq 0)$. As explained in the last section, the massive theory cannot be folded into a boundary theory, so one must solve directly the massive DYB equation, along with the crossing, unitarity and hermiticity constraints. In doing this, we make the assumption that the defect degrees of freedom in the massless and massive cases are the same, and furthermore that the dependence on the defect degrees of freedom is also the same. Thus we look for a solution of the form

$$
T_{\alpha a}^{\beta b}(\theta)=q^{a \alpha / 2} T_{a}^{b}(\theta), \quad a+\alpha=b+\beta,
$$

where $T_{a}^{b}$ is not necessarily the same as the massless $R_{a}^{b}$. For scattering from the left it will be important to take a different ansatz where the phase depends on outgoing quantum numbers:

$$
\widetilde{T}_{a \alpha}^{b \beta}(\theta)=q^{-b \beta / 2} \widetilde{T}_{a}^{b}(\theta)
$$


There are four independent functions for each of $T$ and $\widetilde{T}$ :

$$
\begin{array}{ll}
T_{\alpha \pm}^{\alpha \pm}=q^{ \pm \alpha / 2} P_{ \pm}, & T_{\alpha, \pm}^{\alpha \pm 2, \mp}=q^{ \pm \alpha / 2} Q_{ \pm} \\
\widetilde{T}_{ \pm \alpha}^{ \pm \alpha}=q^{\mp \alpha / 2} \widetilde{P}_{ \pm}, & T_{ \pm, \alpha \mp 2}^{\mp, \alpha}=q^{ \pm \alpha / 2} \widetilde{Q}_{ \pm} .
\end{array}
$$

The DYB equation leads to the equations

$$
\begin{aligned}
& a\left(\theta_{12}\right) P_{ \pm}\left(\theta_{2}\right) Q_{ \pm}\left(\theta_{1}\right)=q b\left(\theta_{12}\right) Q_{ \pm}\left(\theta_{1}\right) P_{ \pm}\left(\theta_{2}\right)+c\left(\theta_{12}\right) P_{ \pm}\left(\theta_{1}\right) Q_{ \pm}\left(\theta_{2}\right) \\
& a\left(\theta_{12}\right) Q_{ \pm}\left(\theta_{2}\right) P_{ \pm}\left(\theta_{1}\right)=q^{-1} b\left(\theta_{12}\right) P_{ \pm}\left(\theta_{1}\right) Q_{ \pm}\left(\theta_{2}\right)+c\left(\theta_{12}\right) Q_{ \pm}\left(\theta_{1}\right) P_{ \pm}\left(\theta_{2}\right) \\
& a\left(\theta_{12}\right) P_{ \pm}\left(\theta_{1}\right) Q_{\mp}\left(\theta_{2}\right)=q^{-1} b\left(\theta_{12}\right) Q_{\mp}\left(\theta_{2}\right) P_{ \pm}\left(\theta_{1}\right)+c\left(\theta_{12}\right) P_{ \pm}\left(\theta_{2}\right) Q_{\mp}\left(\theta_{1}\right) \\
& a\left(\theta_{12}\right) P_{ \pm}\left(\theta_{2}\right) Q_{\mp}\left(\theta_{1}\right)=q b\left(\theta_{12}\right) P_{ \pm}\left(\theta_{2}\right) Q_{\mp}\left(\theta_{1}\right)+c\left(\theta_{12}\right) P_{ \pm}\left(\theta_{1}\right) Q_{\mp}\left(\theta_{2}\right) .
\end{aligned}
$$

The equations satisfied by $\widetilde{P}, \widetilde{Q}$ are the same as above with $q \rightarrow q^{-1}$.

Dividing the equations (5.19) by their left hand sides, and setting $\theta_{2}=0$, one finds that one must satisfy:

$$
(a-q b)\left(a-q^{-1} b\right)=c^{2} .
$$

This requires that $q=-\exp (i \pi \gamma)$ or $-\exp (-i \pi \gamma)$. The two distinct solutions for these distinct values of $q$ correspond to taking left verses right perturbation in (5.1). To match the massless limit of the folded theory, we take $q=-\exp (-i \pi \gamma)$. The general solution to the DYB equation is then

$$
\begin{aligned}
& P_{ \pm}(\theta)=\mu_{ \pm} e^{-\gamma \theta / 2} f(\theta), \quad Q_{ \pm}(\theta)=\nu_{ \pm} e^{\gamma \theta / 2} f(\theta) \\
& \widetilde{P}_{ \pm}(\theta)=\widetilde{\mu}_{ \pm} e^{\gamma \theta / 2} \widetilde{f}(\theta), \quad \widetilde{Q}_{ \pm}(\theta)=\widetilde{\nu}_{ \pm} e^{-\gamma \theta / 2} \widetilde{f}(\theta),
\end{aligned}
$$

where $\mu_{ \pm}, \nu_{ \pm}, \widetilde{\mu}_{ \pm}, \widetilde{\nu}_{ \pm}$are constants, and $f, \widetilde{f}$ are arbitrary.

The hermiticity condition (4.7) reads

$$
\widetilde{P}_{ \pm}(\theta)=P_{ \pm}^{*}(-\theta), \quad \widetilde{Q}_{ \pm}(\theta)=Q_{\mp}^{*}(-\theta) .
$$

The unitarity condition (4.3) then reads

$$
\begin{aligned}
& P_{ \pm}(\theta) P_{ \pm}^{*}(\theta)+Q_{ \pm}(\theta) Q_{ \pm}^{*}(\theta)=1 \\
& P_{ \pm}(\theta) Q_{\mp}^{*}(\theta)+Q_{ \pm}(\theta) P_{\mp}^{*}(\theta)=0 .
\end{aligned}
$$

Finally, the crossing relation (4.4) implies

$$
P_{ \pm}^{*}(\theta)=P_{\mp}(i \pi+\theta), \quad q Q_{\mp}^{*}(\theta)=Q_{ \pm}(i \pi+\theta) .
$$


The minimal solution to (5.23), (5.24) of the form (5.21) is the following:

$$
\begin{aligned}
P_{ \pm}(\theta) & =e^{ \pm i \xi} \frac{e^{-(\gamma \theta+\nu) / 2}}{2 \cosh ((\gamma \theta+\nu-i \pi / 2) / 2)} e^{i \delta(\theta)}, \\
Q_{ \pm}(\theta) & =e^{i \tau_{ \pm}} \frac{e^{(\gamma \theta+\nu) / 2}}{2 \cosh ((\gamma \theta+\nu-i \pi / 2) / 2)} e^{i \delta(\theta)},
\end{aligned}
$$

where

$$
\begin{aligned}
e^{i \delta(\theta)} & =e^{i \pi \gamma / 4} \prod_{l=0}^{\infty} \frac{R_{l}(\gamma \theta+\nu)}{R_{l}(-(\nu+\gamma \theta))} \\
R_{l}(x) & =\frac{\Gamma\left(\frac{3}{4}+l \gamma-\frac{i x}{2 \pi}\right) \Gamma\left(\frac{1}{4}+(l+1) \gamma-\frac{i x}{2 \pi}\right)}{\Gamma\left(\frac{1}{4}+\left(l+\frac{1}{2}\right) \gamma-\frac{i x}{2 \pi}\right) \Gamma\left(\frac{3}{4}+\left(l+\frac{1}{2}\right)-\frac{i x}{2 \pi}\right)}
\end{aligned}
$$

One can add to $\delta(\theta)$ a real function $r(\theta)$ such that

$$
r(\theta)+r(\theta+i \pi)=2 \pi n,
$$

and still obtain a solution. Here is the CDD ambiguity showing itself. The constants $\xi, \tau_{ \pm}$ and $\nu$ are parameters of the solution, with $\tau_{+}+\tau_{-}=n \pi$ where $n$ is an odd integer. By a gauge transformation, we can set $\tau_{+}=\tau_{-}=n \pi / 2$ for any particular odd $\mathrm{n}$. The most significant parameter is $\nu$ which is some unknown function of the dimensionless variables $g^{2} / \lambda, \beta^{2}$.

The solution is minimal in the sense that $\delta(\theta)$ was chosen to bring the solution to within a phase of the massless limit of the folded theory and no more. It is not surprising that we can do so. Once the DYB equations have constrained the phase to be $-\exp ( \pm i \pi \gamma)$, there is little freedom left for a different solution to arise. Though the crossing relationship for a massive boundary field theory,

$$
R_{\bar{a}}^{b}(i \pi-\theta)=S_{a^{\prime} b^{\prime}}^{a b}(2 \theta-i \pi) R_{\bar{b}^{\prime}}^{a^{\prime}}(\theta),
$$

differs significantly from its defect counterpart, its massless limit,

$$
R_{\bar{a}}^{b}(i \pi-\theta)=q^{-a b / 2} R_{\bar{b}}^{a}(\theta),
$$

differs only by a phase. Similarly, unitarity for the boundary theory has the same form as unitarity for the defect theory. 


\subsection{Implementing the Bootstrap}

Here we will use the bootstrap to derive the transmission matrices for the breathers. The breathers in sine-Gordon exist as bound states of solitons. We can express this relation in terms of the Faddeev-Zamolodchikov operators. Let $A_{s}(\theta)$ be the solitons $\left(s_{1}, s_{2}=+/-\right)$ and $A_{b}(\theta)$ be the breathers where $b=1, \ldots, n$ where $\mathrm{n}$ is the maximum integer less than $\gamma$. Then we have

$$
f_{s_{1} s_{2}}^{b} A_{b}(\theta)=A_{s_{1}}\left(\theta+i \bar{u}_{s_{1} \bar{b}}^{\bar{s}_{2}}\right) A_{s_{2}}\left(\theta-i \bar{u}_{s_{2} \bar{b}}^{\bar{s}_{1}}\right)
$$

where $\bar{s}=-s$ is the charge conjugate of $s$. The $\bar{u}$ 's are given by

$$
\bar{u}_{s b}^{s^{\prime}}=\pi-u_{s b}^{s^{\prime}}
$$

where $i u_{s b}^{s^{\prime}}$ is the location of the soliton pole in the soliton-breather scattering matrix. Here we have

$$
u_{ \pm b}^{ \pm}=\frac{\pi}{2}+\frac{n \pi}{2 \lambda}
$$

The $f$ 's are related to the residues of the poles in the soliton-anti-soliton scattering matrices. If $u_{+-}^{b}$ is the location of the pole in $S_{+-}^{ \pm \mp}$, indicative of breather b, the $f_{\mp \pm}^{b}$ are defined via

$$
S_{+-}^{ \pm \mp}(\theta) \sim i \frac{f_{+-}^{b} f_{b}^{ \pm \mp}}{\theta-i u_{+-}^{b}},
$$

where there is no summation on $b . f_{s_{1} s_{2}}^{b}$ thus represents the probability amplitude that the bound state forms from a soliton pair. It is then easy to show that

$$
\begin{aligned}
f_{+-}^{b} f_{b}^{+-} & =(-1)^{b} S_{o} \\
f_{-+}^{b} f_{b}^{+-} & =S_{o} \\
f_{ \pm \pm}^{b} & =0
\end{aligned}
$$

where $S_{o}$ is some constant. Hence $f_{+-}^{b} / f_{-+}^{b}=(-1)^{b}$. This last relation is all we will need for the computation of the breather transmission matrices.

The transmission bootstrap equation is

$$
f_{s_{1} s_{2}}^{b} T_{\alpha b}^{\gamma d}(\theta)=f_{g_{3} g_{2}}^{d} T_{\alpha s_{2}}^{\beta g_{2}}\left(\theta-i \bar{u}_{s_{2} \bar{b}}^{\bar{s}_{1}}\right) T_{\beta s_{1}}^{\gamma g_{3}}\left(\theta+i \bar{u}_{s_{1} \bar{b}}^{\bar{s}_{2}}\right)
$$

This is derived through the consistency of (5.30) scattering through the defect. In fact it is more general than indicated: $b$ need not be a breather and $s_{1}, s_{2}$ need not be solitons for the equation to hold. 
Now taking $b$ to be some breather and $s_{1}=+, s_{2}=-$, we can write an explicit expression for the breather transmission matrix:

$$
T_{\alpha b}^{\alpha b}(\theta)=P_{-}\left(\theta-i \bar{u}_{-b}^{-}\right) P_{+}\left(\theta+i \bar{u}_{+b}^{+}\right)+(-1)^{b} q^{-1} Q_{-}\left(\theta-i \bar{u}_{-b}^{-}\right) Q_{+}\left(\theta+i \bar{u}_{+b}^{+}\right) .
$$

As expected, the breather scattering matrix does not depend on the parameter $\alpha$. With this, we can explicitly check if $T_{\alpha b}^{\alpha b}(\theta)$ satisfies crossing and unitarity. We find that both

$$
\begin{aligned}
& T_{\alpha b}^{* \alpha b}(\theta)=T_{\alpha b}^{\alpha b}(i \pi+\theta) \\
& T_{\alpha b}^{\alpha b}(\theta) T_{\alpha b}^{* \alpha b}(\theta)=1
\end{aligned}
$$

hold without putting any further constraints on $\delta(\theta)$ in (5.25) and (5.26) .

\section{The Free Fermion Point}

In this section we will consider the chiral defect SG theory at the free fermion point $\beta^{2}=4 \pi$, where we can derive the defect scattering matrices from an action. For the reasons indicated at the end of section 5.1, we expect that this theory will exhibit reflection in addition to transmission in the massive case $\lambda \neq 0$, and this is what we find. In the massless limit we recover the purely transmitting S-matrix of section 5.2. This suggests the massive transmission S-matrix of section 5 only has significance in its massless limit, since it was obtained assuming only transmission. However we have not absolutely ruled out that an alternative massive fermion action may still be purely transmitting with massive transmission given by $T_{\alpha a}^{\beta b}\left(\theta, \beta^{2}=4 \pi\right)$ of the last section.

In terms of fermions, the action (4.1) can be expressed as

$$
\begin{aligned}
& S=S_{\mathrm{bulk}}+S_{\text {free }}+S_{\mathrm{defect}} \\
& =\frac{1}{8 \pi} \sum_{i= \pm} \int d x d t \psi_{+}^{(i)} \partial_{\bar{z}} \psi_{-}^{(i)}+\psi_{-}^{(i)} \partial_{\bar{z}} \psi_{+}^{(i)}+\bar{\psi}_{+}^{(i)} \partial_{z} \bar{\psi}_{-}^{(i)}+\bar{\psi}_{-}^{(i)} \partial_{z} \bar{\psi}_{+}^{(i)} \\
& +2 i m\left(\bar{\psi}_{+}^{(i)} \psi_{-}^{(i)}-\psi_{+}^{(i)} \bar{\psi}_{-}^{(i)}\right) \\
& -\frac{i}{8 \pi} \int d t \psi_{-}^{(+)} \psi_{+}^{(-)}+\bar{\psi}_{+}^{(-)} \bar{\psi}_{-}^{(+)}+\psi_{+}^{(+)} \psi_{-}^{(-)}+\bar{\psi}_{-}^{(-)} \bar{\psi}_{+}^{(+)} \\
& -\frac{g}{4 \pi} \int d t \psi_{+}^{(-)} a_{-}+\psi_{+}^{(+)} a_{+}+a_{+} \psi_{-}^{(-)}+a_{-} \psi_{-}^{(+)}-\frac{1}{2 \pi} \int d t a_{+} \partial_{t} a_{-} .
\end{aligned}
$$

Here we have used the standard map between fermions and bosons at $\beta^{2}=4 \pi: \psi_{ \pm}=$ $\exp \left(i \phi_{L}\right)$ and $\bar{\psi}_{ \pm}=\exp \left(\mp \phi_{R}\right)$ [11] [12]. The bulk term represents, locally, the massive 
sine-Gordon action. However, we have divided it into two parts: one for the theory in the region $(x>0)$ (terms with the superscript $(+))$ and one for the region $(x<0)$ (terms with the superscript $(-))$. Because the two regions $(+)$ and $(-)$ are decoupled, we need to add to the action a term which insures continuity, i.e.

$$
\psi_{ \pm}^{(-)}-\psi_{ \pm}^{(+)}=\bar{\psi}_{ \pm}^{(-)}-\bar{\psi}_{ \pm}^{(+)}=0
$$

in the absence of a defect $(g=0)$. This term is $S_{\text {free }}$. The defect term is constructed in analogy to what was done in [13]. There Fermi fields were coupled to fermionic defect modes, $a_{ \pm}$. Although the origin of the $a_{ \pm}$is more obscure here, we must have the same thing, if only to insure the action is bosonic. The specific choice of mixing and matching the $a_{ \pm}$'s and the $\psi_{ \pm}$'s is determined by unitarity, by the need to have terms which both violate and preserve $\mathrm{U}(1)$ charge, and in part by trial and error (so as to obtain, in the massless limit, the solution of the DYB equations in section 5). The defect degrees of freedom introduced in section 5 can be understood as arising from the zero modes $a_{ \pm}$. To derive the scattering matrices for this action, we begin by writing down the equations of motion. Varying with respect to $\psi_{ \pm}^{(+),(-)}, \bar{\psi}_{ \pm}^{(+),(-)}$, and $a_{ \pm}$, and then eliminating the $a_{ \pm}$ modes, leaves us with three equations, all evaluated at $x=0$ :

$$
\begin{aligned}
& 0=i \partial_{t}\left(\psi_{+}^{(+)}-\psi_{+}^{(-)}\right)+g^{2}\left(\psi_{+}^{(+)}-\psi_{-}^{(-)}\right) \\
& 0=\psi_{-}^{(+)}-\psi_{-}^{(-)}+\psi_{+}^{(+)}-\psi_{+}^{(-)} \\
& 0=\bar{\psi}_{ \pm}^{(+)}-\bar{\psi}_{ \pm}^{(-)} .
\end{aligned}
$$

The first two equations describe interactions among the chiral fermions. The last equation is nothing more than the continuity of the anti-chiral fermions.

To determine the scattering matrices, we employ the following mode expansions for the fermions:

$$
\begin{aligned}
& \psi_{+}=\sqrt{m} \int_{-\infty}^{\infty} \frac{d \theta}{2 \pi i} e^{-\theta / 2}\left(A_{-}(\theta) e^{-m\left(z e^{-\theta}+\bar{z} e^{\theta}\right)}-A_{+}^{\dagger}(\theta) e^{m\left(z e^{-\theta}+\bar{z} e^{\theta}\right)}\right) \\
& \bar{\psi}_{+}=-i \sqrt{m} \int_{-\infty}^{\infty} \frac{d \theta}{2 \pi i} e^{\theta / 2}\left(A_{-}(\theta) e^{-m\left(z e^{-\theta}+\bar{z} e^{\theta}\right)}+A_{+}^{\dagger}(\theta) e^{m\left(z e^{-\theta}+\bar{z} e^{\theta}\right)}\right)
\end{aligned}
$$

where $\psi_{-}=\psi_{+}^{\dagger}$ and $\bar{\psi}_{-}=\bar{\psi}_{+}^{\dagger}$. The A's satisfy the following anti-commutation relations:

$$
\left\{A_{ \pm}(\theta), A_{\mp}^{\dagger}\left(\theta^{\prime}\right)\right\}=4 \pi^{2} \delta\left(\theta-\theta^{\prime}\right) .
$$


We now substitute the expansions into the equations of motion, Fourier decompose, and express the equations in the form:

$$
\begin{aligned}
& \mathbf{D} A_{a}^{\dagger(+)}(\theta)=T_{a}^{b}(\theta) A_{b}^{\dagger(-)}(\theta) \mathbf{D}+R_{a}^{b}(\theta) \mathbf{D} A_{b}^{\dagger(+)}(-\theta) \\
& A_{a}^{\dagger(-)}(\theta) \mathbf{D}=\widetilde{T}_{a}^{b}(-\theta) \mathbf{D} A_{b}^{\dagger(+)}(-\theta)+\widetilde{R}_{a}^{b}(-\theta) A_{b}^{\dagger(-)}(-\theta) \mathbf{D},
\end{aligned}
$$

or upon expanding out

$$
\begin{aligned}
& \mathbf{D} A_{ \pm}^{\dagger(+)}(\theta)= P_{ \pm}(\theta) A_{ \pm}^{\dagger(-)}(\theta) \mathbf{D}+Q_{ \pm}(\theta) A_{\mp}^{\dagger(-)}(\theta) \mathbf{D}+ \\
& M_{ \pm}(\theta) \mathbf{D} A_{ \pm}^{\dagger(+)}(-\theta)+N_{ \pm}(\theta) \mathbf{D} A_{\mp}^{\dagger(+)}(-\theta) \\
& A_{ \pm}^{\dagger(-)}(\theta) \mathbf{D}=\widetilde{P}_{ \pm}(-\theta) \mathbf{D} A_{ \pm}^{\dagger(+)}(\theta)+\widetilde{Q}_{ \pm}(-\theta) \mathbf{D} A_{\mp}^{\dagger(+)}(\theta)+ \\
& \widetilde{M}_{ \pm}(-\theta) A_{ \pm}^{\dagger(-)}(-\theta) \mathbf{D}+\widetilde{N}_{ \pm}(-\theta) A_{\mp}^{\dagger(-)}(-\theta) \mathbf{D} .
\end{aligned}
$$

These equations define the S-matrices for the theory. $P_{ \pm}$and $Q_{ \pm}$represent transmission across the defect while $M_{ \pm}$and $N_{ \pm}$represent reflection from the defect. We find the matrices to be

$$
\begin{aligned}
& P_{ \pm}(\theta)=\widetilde{P}_{ \pm}^{*}(-\theta)=\frac{f(\theta)+e^{2 \theta}-1}{f(\theta)} ; \\
& Q_{ \pm}(\theta)=\widetilde{Q}_{ \pm}^{*}(-\theta)=\frac{1-e^{2 \theta}}{f(\theta)} ; \\
& M_{ \pm}(\theta)=\widetilde{M}_{ \pm}^{*}(-\theta)=-\frac{2 \sinh (\theta)}{f(\theta)} ; \\
& N_{ \pm}(\theta)=\widetilde{N}_{ \pm}^{*}(-\theta)=\frac{2 \sinh (\theta)}{f(\theta)}
\end{aligned}
$$

where

$$
f(\theta)=-\frac{4 i m}{g^{2}} \cosh (\theta) \sinh ^{2}(\theta)+2 \sinh (2 \theta) .
$$

The matrices do not differentiate between U(1) charge because of our choice of $S_{\text {free. }}$ If we wrote $S_{\text {free }}$ as

$$
S_{\text {free }}=-\frac{i}{8 \pi} \int d t e^{i \alpha} \psi_{-}^{(+)} \psi_{+}^{(-)}+e^{i \beta} \bar{\psi}_{-}^{(-)} \bar{\psi}_{-}^{(+)}+e^{-i \alpha} \psi_{+}^{(+)} \psi_{-}^{(-)}+e^{-i \beta} \bar{\psi}_{-}^{(-)} \bar{\psi}_{+}^{(+)}
$$

the \pm matrices would be distinguished by a phase.

Because the theory is both reflecting and transmitting, crossing and unitarity are altered. Unitarity becomes

$$
\begin{aligned}
& 1=T_{a}^{b}(\theta) \widetilde{T}_{b}^{c}(-\theta)+R_{a}^{b}(\theta) R_{b}^{c}(-\theta) \\
& 0=T_{a}^{b}(\theta) \widetilde{R}_{b}^{c}(-\theta)+R_{a}^{b}(\theta) T_{b}^{c}(-\theta),
\end{aligned}
$$


in addition to two more equations where $\widetilde{T}(-\theta)$ and $\widetilde{R}(-\theta)$ are interchanged with $T(\theta)$ and $R(\theta)$. Crossing keeps its transmission component in (4.4) and adds a reflection piece:

$$
\begin{aligned}
& R_{\bar{a}}^{b}(\theta)=-R_{\frac{a}{b}}^{a}(i \pi-\theta) ; \\
& \widetilde{R}_{\bar{a}}^{b}(\theta)=-\widetilde{R}_{\bar{b}}^{a}(i \pi-\theta) .
\end{aligned}
$$

One can easily check that the scattering matrices derived above satisfy these relations.

As promised, the massless limit of these matrices match the scattering matrices in 5.34. To find the massless limit of the matrices for scattering of the left movers from right to left take $\theta \rightarrow \theta_{L}-\alpha$ and let $\alpha \rightarrow \infty, m \rightarrow 0$ while holding $\mu=m e^{\alpha}$ constant. We so find

$$
\begin{aligned}
P_{ \pm}\left(\theta_{L}\right) & =\frac{e^{i \pi / 4} e^{-\left(\theta_{L}+\nu\right) / 2}}{2 \cosh \left(\left(\theta_{L}+\nu-i \pi / 2\right) / 2\right)}, \\
Q_{ \pm}\left(\theta_{L}\right) & =\frac{e^{-i \pi / 4} e^{\left(\theta_{L}+\nu\right) / 2}}{2 \cosh \left(\left(\theta_{L}+\nu-i \pi / 2\right) / 2\right)}, \\
M_{ \pm}\left(\theta_{L}\right) & =N_{ \pm}\left(\theta_{L}\right)=0 .
\end{aligned}
$$

Here we have identified $\nu$ with $-\log \left(\mu / 2 g^{2}\right)$. To obtain an exact match with section 5 , we need to fix our gauge by setting $\tau_{+}=\tau_{-}=-\pi / 2$.

\section{Ising Model}

Having considered a chiral defect theory for a Dirac fermion, it is natural to also treat a Majoranna fermion, i.e. the fermionic representation of the Ising model. As with the Dirac fermion, we find a massive Majoranna fermion has both transmission and reflection S-matrices. And again, we find that in the massless limit, the reflection matrices vanish.

Defect lines in Ising models have been considered before ([14 [15] [16]), but in a different form. Previously, defects as perturbations in the energy were studied. In terms of fermions, a energy perturbation is represented by $\psi \bar{\psi}$. Here instead we consider defects as perturbations in a single $\psi$. Whereas energy perturbations have ready interpretations in terms of spin operators in a lattice formulation, a chiral perturbation does not. Its physical interpretation comes only in folding the theory onto boundary Ising.

The action for the Ising model can be expressed in the same form as (6.1),

$$
\begin{aligned}
S & =S_{\text {bulk }}+S_{\text {free }}+S_{\text {defect }} \\
= & \frac{1}{8 \pi} \sum_{i= \pm} \int d x d t\left(\psi^{(i)} \partial_{\bar{z}} \psi^{(i)}+\bar{\psi}^{(i)} \partial_{z} \bar{\psi}^{(i)}+2 i m \psi^{(i)} \bar{\psi}^{(i)}\right) \\
& -\frac{i}{8 \pi} \int d t\left(\psi^{(+)} \psi^{(-)}+\bar{\psi}^{(+)} \bar{\psi}^{(-)}\right) \\
& +\frac{i g}{4 \pi} \int d t\left(a \psi^{(+)}-\psi^{(-)} a\right)-\frac{1}{4 \pi} \int d t a \partial_{t} a .
\end{aligned}
$$


With the Dirac fermion, the defect modes, $a_{ \pm}$, were indexed to reflect the $\mathrm{U}(1)$ charge carried by the fermionic fields. Here there fields have no charge and so the defect mode, $a$, goes unstructured.

As before, we determine the scattering matrices by varying the action and writing down the equations of motion at $x=0$ :

$$
\begin{aligned}
& 0=\partial_{t}\left(\psi^{(+)}-\psi^{(-)}\right)-i g^{2}\left(\psi^{(+)}+\psi^{(-)}\right) \\
& 0=\bar{\psi}^{(+)}-\bar{\psi}^{(-)}
\end{aligned}
$$

The first equation describes the interaction of the chiral fermion, while the second equation simply enforces the continuity of the anti-chiral fermion across the defect line. We employ the following mode expansions:

$$
\begin{aligned}
& \psi=\sqrt{m} \int_{-\infty}^{\infty} \frac{d \theta}{2 \pi i} e^{-\theta / 2}\left(A(\theta) e^{-m\left(z e^{-\theta}+\bar{z} e^{\theta}\right)}-A^{\dagger}(\theta) e^{m\left(z e^{-\theta}+\bar{z} e^{\theta}\right)}\right) \\
& \bar{\psi}=-i \sqrt{m} \int_{-\infty}^{\infty} \frac{d \theta}{2 \pi i} e^{\theta / 2}\left(A(\theta) e^{-m\left(z e^{-\theta}+\bar{z} e^{\theta}\right)}+A^{\dagger}(\theta) e^{m\left(z e^{-\theta}+\bar{z} e^{\theta}\right)}\right)
\end{aligned}
$$

Here $\left\{A(\theta), A^{\dagger}\left(\theta^{\prime}\right)\right\}=4 \pi^{2} \delta\left(\theta-\theta^{\prime}\right)$. Substituting these expansions into the equations of motion, and reducing them to the form

$$
\begin{aligned}
& \mathbf{D} A^{\dagger(+)}(\theta)=T(\theta) A^{\dagger(-)} \mathbf{D}+R(\theta) \mathbf{D} A^{\dagger(+)}(-\theta) \\
& A^{\dagger(-)}(\theta) \mathbf{D}=\widetilde{T}(-\theta) \mathbf{D} A^{\dagger(+)}(\theta)+\widetilde{R}(-\theta) A^{\dagger(-)}(-\theta) \mathbf{D},
\end{aligned}
$$

leads to the result

$$
\begin{aligned}
& T(\theta)=\widetilde{T}^{*}(-\theta)=\frac{m \cosh (\theta)+i g^{2}}{m \cosh (\theta)+i g^{2} \operatorname{coth}(\theta)} \\
& R(\theta)=\widetilde{R}^{*}(-\theta)=-\frac{i g^{2}}{m \cosh (\theta) \sinh (\theta)+i g^{2} \cosh (\theta)}
\end{aligned}
$$

In the massless limit, $R(\theta)$ and $T(\theta)$ become

$$
\begin{aligned}
T\left(\theta_{L}\right) & =\frac{\mu e^{-\theta_{L}}+i 2 g^{2}}{\mu e^{-\theta_{L}}-i 2 g^{2}}, \\
R\left(\theta_{L}\right) & =0 .
\end{aligned}
$$

As expected the reflection amplitude, $R\left(\theta_{L}\right)$, vanishes. The massless transmission matrix can be compared with the massless limit of the reflection matrix, $R_{B}$, derived in [2] for boundary Ising. In this case we find

$$
R_{B}\left(\theta_{L}\right)=-i \frac{\mu e^{-\theta_{L}}+i h^{2}}{\mu e^{-\theta_{L}}-i h^{2}},
$$


where $h$ is the boundary magnetic field. By identifying $2 g^{2}$ with $h^{2}$, a phase is the sole difference between the two massless limits. This phase arises from the difference between the boundary and defect crossing relations.

In this case unitarity takes the form,

$$
\begin{aligned}
& 1=T(\theta) \widetilde{T}(-\theta)+R(\theta) R(-\theta) \\
& 0=T(\theta) \widetilde{R}(-\theta)+R(\theta) T(-\theta)
\end{aligned}
$$

while crossing demands,

$$
T(\theta)=\widetilde{T}(i \pi-\theta), \quad R(\theta)=-R(i \pi-\theta), \quad \widetilde{R}(\theta)=-\widetilde{R}(i \pi-\theta) .
$$

It easily seen that the scattering amplitudes satisfy these equations.

\section{Conclusions}

We have outlined a general approach to integrable chiral defect theories, and have applied this to a chiral defect perturbation of the sine-Gordon theory.

A nice feature of purely transmitting theories of defects is that they allow one to consider multiple defects rather easily, and consequently a finite density of impurities. We hope to turn to this in the future.

\section{Acknowledgments}

We would like to thank F. Lesage, A. Ludwig, and H. Saleur for useful discussions. This work is supported both by the National Science Foundation, in part through the National Young Investigator Program, and under Grant No. PHY94-07194, and the National Science and Engineering Research Council of Canada, through an NSERC PGS B fellowship. 


\section{References}

[1] A. Zamolodchikov, Int. Journ. Mod. Phys. A4 (1989) 4235.

[2] S. Ghoshal and A. Zamolodchikov, Int. J. Mod. Phys. A 9 (1994) 3841.

[3] G. Delfino, G. Mussardo, P. Simonetti, Nucl. Phys. B432 (1994) 518.

[4] J. L. Cardy, Nucl. Phys. B275 (1986) 200-218.

[5] M. Oshikawa and I. Affleck, Phys. Rev. Lett. 77 (1996) 2604.

[6] E. Wong and I. Affleck, Nucl. Phys B417 (1994) 403.

[7] P. Fendley, A.W.W. Ludwig, H. Saleur, Phys. Rev. Lett. (1995) 3005.

[8] A. B. Zamolodchikov and Al. B. Zamolodchikov, Ann. Phys. 120 (1979) 253.

[9] P. Fendley, H. Saleur, N. P. Warner, Nucl. Phys. B (1994) 577.

[10] P. Fendley, F. Lesage and H. Saleur, A Unified Framework for the Kondo Problem and for an Impurity in a Luttinger Liquid, cond-mat/9510055.

[11] S. Coleman, Phys. Rev. D 11 (1975) 2088.

[12] S. Mandelstam, Phys. Rev. D 113026 (1975).

[13] M. Ameduri, R. Konik, and A. LeClair, Phys. Lett. B 354 (1995) 376.

[14] R. Bariev, Sov. Phys. JETP 50 (1979) 613.

[15] B. McCoy and J. H. H. Perk, Phys. Rev. Lett. 44 (1980) 840.

[16] B. McCoy and J. H. H. Perk, The Riemann Problem, Complete Integrability and Arithmetic Applications 925 (1982) 12. 